\title{
SOME REMARKS ON HERMITIAN MANIFOLDS SATISFYING KÄHLER-LIKE CONDITIONS
}

\author{
ANNA FINO AND NICOLETTA TARDINI
}

\begin{abstract}
We study Hermitian metrics whose Bismut connection $\nabla^{B}$ satisfies the first Bianchi identity in relation to the SKT condition and the parallelism of the torsion of the Bimut connection. We obtain a characterization of complex surfaces admitting Hermitian metrics whose Bismut connection satisfy the first Bianchi identity and the condition $R^{B}(x, y, z, w)=R^{B}(J x, J y, z, w)$, for every tangent vectors $x, y, z, w$, in terms of Vaisman metrics. These conditions, also called Bismut Kähler-like, have been recently studied in [4] 30] 28]. Using the characterization of SKT almost abelian Lie groups in 5, we construct new examples of Hermitian manifolds satisfying the Bismut Kähler-like condition. Moreover, we prove some results in relation to the pluriclosed flow on complex surfaces and on almost abelian Lie groups. In particular, we show that, if the initial metric has constant scalar curvature, then the pluriclosed flow preserves the Vaisman condition on complex surfaces.
\end{abstract}

\section{INTRODUCTION}

Given a Hermitian manifold $(X, J, g)$, the Bismut connection $\nabla^{B}$ is the unique connection on $X$ that is Hermitian (i.e. such that $\nabla^{B} g=0$ and $\nabla^{B} J=0$ ) and has totally skew-symmetric torsion tensor (cf. [8]). If the torsion 3-form of $\nabla^{B}$ is closed, the Hermitian metric $g$ is called $S K T$ or pluriclosed. Examples of SKT manifolds are given by Lie groups (and its compact quotients) endowed with left-invariant SKT Hermitian structures (see for instance [12, 24], 11]). A characterization of almost abelian Lie groups, i.e. Lie groups whose Lie algebra has a codimensionone abelian ideal, admitting left-invariant SKT metrics has been recently obtained in $[5]$.

In 27] the authors studied Hermitian metrics whose Levi-Civita and Chern connection have curvature tensors satisfying all the symmetry conditions of a Kähler metric. Hermitian metrics with the Bismut connection being "Kähler-like", namely, satisfying the first Bianchi identity and the condition $R^{B}(x, y, z, w)=$ $R^{B}(J x, J y, z, w)$, for every tangent vectors $x, y, z, w$, have been studied, in [4], investigating this property on 6-dimensional solvmanifolds with holomorphically trivial canonical bundle.

In 30] Zhao and Zheng show that if the curvature tensor of the Bismut connection satisfies the symmetry conditions

$$
R^{B}(x, y, z, w)=R^{B}(z, y, x, w), \quad R^{B}(x, y, J z, J w)=R^{B}(x, y, z, w)
$$

for any tangent vectors $x, y, z, w$ in $X$, then the Hermitian metric must be SKT. In [28, a classification for compact non-Kähler Hermitian manifolds satisfying (1) in complex dimension 3 and those with degenerate torsion in higher dimensions is given.

2010 Mathematics Subject Classification. 53C55; 53C05; 22E25; 53C30; 53C44.

Key words and phrases. Bismut connection, curvature, Hermitian metric. 
An evolution equation of SKT metrics is given by the pluriclosed flow, introduced by Streets and Tian in [19]. In recent years the pluriclosed flow has been an active subject of study, and already many regularity and convergence results have been proved in [20, 21]. A natural question is to see if the Bismut Kähler-like condition is preserved by the flow.

In this paper we study Hermitian metrics whose Bismut connection $\nabla^{B}$ satisfies the first Bianchi identity in relation to the SKT condition and the parallelism of the torsion of the Bismut connection. In particular, as a consequence of Theorem 3.1 and Theorem 3.2 we show that, if $X$ is a complex manifold and $g$ is a Hermitian metric such that the Bismut connection satisfies the first Bianchi identity, then,

$$
\nabla^{B} T^{B}=0 \quad \Longleftrightarrow \quad g \text { is SKT. }
$$

Moreover, we show in Proposition 3.11 that the existence of these metrics is not open under small deformations of the complex structure.

Specializing to complex dimension 2 we are able to characterize these metrics as follows

Theorem A. Let $X$ be a complex surface and $g$ be a Hermitian metric. Then, $g$ is Vaisman if and only if $g$ is a SKT metric and the Bismut connection satisfies the first Bianchi identity.

Where we recall that a Vaisman metric $\omega$ on a complex manifold $X$ is a Hermitian metric satisfying $d \omega=\theta \wedge \omega$ for some $d$-closed 1-form $\theta$ with $\nabla^{L C} \theta=0$.

In [7] a generalization of Vaisman metrics, called metrics with Lee potential, is introduced, and in subsection 3.1 we study this condition in relation with the SKT condition.

In Section 4 we construct new examples of Hermitian manifolds satisfying the Bismut Kähler-like condition, using the characterization of SKT simply connected almost abelian Lie groups. In order to do this we compute explicitly the components of the Bismut connection. Moreover, we give conditions on the structure equations of almost abelian Lie groups in order to have Kähler and flat Hermitian metrics.

In the last Section we study the Bismut Kähler-like condition in relation to the pluriclosed flow, discussing its behavior on complex dimension 2 and on almost abelian Lie groups. In particular, we prove the following

Theorem B. Let $X$ be a compact complex surface admitting a Vaisman metric $\omega_{0}$ with constant scalar curvature, then the pluriclosed flow starting with $\omega_{0}$ preserves the Vaisman condition.

Acknowledgments. The authors would like to thank Luigi Vezzoni and Mihaela Pilca for useful discussions and suggestions. The authors would like to thank also Quanting Zhao and Fangyang Zheng for useful comments. The authors are grateful to Jeffrey Streets for pointing out an inaccuracy in the first version of Theorem B. The authors are supported by Project PRIN 2017 "Real and complex manifolds: Topology, Geometry and Holomorphic Dynamics", by project SIR 2014 AnHyC "Analytic aspects in complex and hypercomplex geometry" code RBSI14DYEB, and by GNSAGA of INdAM.

\section{Preliminaries}

Let $(X, J)$ be a complex manifold of complex dimension $n$ and let $g$ be a Hermitian metric on $X$ with associated fundamental form $\omega(\cdot, \cdot)=g(\cdot, J \cdot)$. An affine connection is called Hermitian if it preserves the metric $g$ and the complex structure $J$. In particular, Gauduchon in [14] proved that there exists an affine line $\left\{\nabla^{t}\right\}_{t \in \mathbb{R}}$ of canonical Hermitian connections, passing through the Chern connection and the 
Bismut connection; these connections are completely determined by their torsion. Let $\nabla$ be a Hermitian connection and $T(x, y)=\nabla_{x} y-\nabla_{y} x-[x, y]$ be its torsion, we denote with the same symbol

$$
T(x, y, z):=g(T(x, y), z) .
$$

Then the Chern connection $\nabla^{C h}$ is the unique Hermitian connection whose torsion has trivial $(1,1)$-component and the Bismut connection (also called Strominger connection) $\nabla^{B}$ is the unique Hermitian connection with totally skew-symmetric torsion. In particular, the torsion of the Bismut connection satisfies

$$
T^{B}(x, y, z)=d^{c} \omega(x, y, z)
$$

where $d^{c}=-J^{-1} d J$.

A Hermitian metric $\omega$ is called strong Kähler with torsion (SKT for brevity) or pluriclosed if $T^{B}$ is a closed 3-form, namely $d T^{B}=0$, or equivalently $d d^{c} \omega=0$.

Recall that the trace of the torsion of the Chern connection is equal to the Lee form of $\omega$ (cf. [13]), that is the 1 -form defined by

$$
\theta=J d^{*} \omega
$$

where $d^{*}$ is the adjoint of the exterior derivative $d$ with respect to $\omega$, or equivalently $\theta$ is the unique 1 -form satisfying

$$
d \omega^{n-1}=\theta \wedge \omega^{n-1} .
$$

A Hermitian metric $\omega$ is called Gauduchon if $d d^{c} \omega^{n-1}=0$, or equivalently $d^{*} \theta=0$. In particular, in dimension 2 Gauduchon and SKT metrics coincide. We recall the following

Definition 2.1. A Hermitian metric $\omega$ on $X$ is called locally conformally Kähler (lck for brevity) if

$$
d \omega=\alpha \wedge \omega
$$

where $\alpha$ is a d-closed 1-form. In particular, $\alpha=\frac{1}{n-1} \theta$ and $\theta$ is $d$-closed. A locally conformally Kähler metric is called Vaisman if the Lee form is parallel with respect to the Levi-Civita connection $\nabla^{L C}$, namely

$$
\nabla^{L C} \theta=0 \text {. }
$$

In particular, it is immediate to see that Vaisman metrics are Gauduchon and the norm of the Lee form $|\theta|$ with respect to $\omega$ is constant.

The Chern and Bismut connections connections are related to the Levi-Civita connection $\nabla^{L C}$ by

$$
\begin{gathered}
g\left(\nabla_{x}^{B} y, z\right)=g\left(\nabla_{x}^{L C} y, z\right)+\frac{1}{2} d^{c} \omega(x, y, z), \\
g\left(\nabla_{x}^{C h} y, z\right)=g\left(\nabla_{x}^{L C} y, z\right)+\frac{1}{2} d \omega(J x, y, z) .
\end{gathered}
$$

If we denote with

$$
\begin{aligned}
R(x, y) z & =\nabla_{x} \nabla_{y} z-\nabla_{y} \nabla_{x} z-\nabla_{[x, y]} z, \\
R(x, y, z, u) & =g(R(x, y) z, u),
\end{aligned}
$$

the curvature tensor of type $(1,3)$ and $(0,4)$, respectively, of a connection $\nabla$ then we have the following identities involving the torsion and the curvature of the Bismut connection (cf. [15]) which will be useful in the following (cf. [15, Formulas (3.20), $(3.21)])$

$$
\begin{aligned}
d T^{B}(x, y, z, u)= & \sigma_{x, y, z}\left\{\left(\nabla_{x}^{B} T^{B}\right)(y, z, u)+2 g\left(T^{B}(x, y), T^{B}(z, u)\right)\right\} \\
& -\left(\nabla_{u}^{B} T^{B}\right)(x, y, z)
\end{aligned}
$$




$$
\begin{aligned}
\sigma_{x, y, z} R^{B}(x, y, z, u)= & d T^{B}(x, y, z, u)+\left(\nabla_{u}^{B} T^{B}\right)(x, y, z) \\
& -\sigma_{x, y, z} g\left(T^{B}(x, y), T^{B}(z, u)\right) .
\end{aligned}
$$

In particular, this shows that the Bismut connection does not satisfy the first Bianchi identity in general. We recall the following definition (cf. 4, Definition 4], [27, [30])

Definition 2.2. The Bismut connection $\nabla^{B}$ is called Kähler-like if it satisfies the first Bianchi identity

$$
\sigma_{x, y, z} R^{B}(x, y, z)=0
$$

and the type condition

$$
R^{B}(x, y, z, w)=R^{B}(J x, J y, z, w) .
$$

In 4 the authors study these two conditions for the canonical connections considered by Gauduchon on 6-dimensional solvmanifolds with invariant complex structures, trivial canonical bundle and invariant Hermitian metrics.

In 30] Zhao and Zheng show that if the curvature tensor of the Bismut connection satisfies the symmetry conditions

$$
R^{B}(x, y, z, w)=R^{B}(z, y, x, w), \quad R^{B}(x, y, J z, J w)=R^{B}(x, y, z, w)
$$

for any tangent vectors $x, y, z, w$ in $X$, then the Hermitian metric must be SKT. We will show that the previous condition for the curvature of the Bismut connection to be symmetric when the first and the third position are interchanged is stronger than first Bianchi identity. In fact, in a private communication Zhao and Zheng showed that those two conditions are equivalent to the vanishing of the curvature $R^{B}$.

\section{Bismut KäHER-LIKE CONDITION}

In this section we investigate the properties (4) and (5) in the definition of Bismut Kähler like Hermitian metrics. In particular, we focus on the relations between the first Bianchi identity for the Bismut connection, the SKT condition and the parallelism of the torsion of the Bismut connection, specializing then our considerations to dimension 2, giving a characterization for Vaisman metrics.

Theorem 3.1. Let $X$ be a complex manifold with a SKT Hermitian metric $g$ such that the Bismut connection satisfies the first Bianchi identity. Then,

$$
\nabla^{B} T^{B}=0 .
$$

Proof. By hypothesis, $d T^{B}=0$ and the first Bianchi-identity holds, hence by Formula (3) one has that for any tangent vectors $x, y, z, u$,

$$
\left(\nabla_{u} T^{B}\right)(x, y, z)=\sigma_{x, y, z} g\left(T^{B}(x, y), T^{B}(z, u)\right)
$$

and by Formula (2)

$$
\sigma_{x, y, z}\left(\nabla_{x}^{B} T^{B}\right)(y, z, u)+\sigma_{x, y, z} 2 g\left(T^{B}(x, y), T^{B}(z, u)\right)-\left(\nabla_{u}^{B} T^{B}\right)(x, y, z)=0 .
$$

Hence, one gets

$$
\sigma_{x, y, z}\left(\nabla_{x}^{B} T^{B}\right)(y, z, u)=-\left(\nabla_{u}^{B} T^{B}\right)(x, y, z) .
$$

Now notice that both sides of the equality are tensorial and on the left hand side the expression is symmetric in $x, y, z$ while on the right hand side is antisymmetric in $x, y, z$. Therefore in order to be equal they must vanish. Hence, $\nabla^{B} T^{B}=0$.

Theorem 3.2. Let $X$ be a complex manifold and let $g$ be a Hermitian metric such that $\nabla^{B} T^{B}=0$. Then, the Bismut connection satisfies the first Bianchi identity if and only if $g$ is SKT. 
Proof. If $\nabla^{B} T^{B}=0$ then by Formula (2)

$$
d T^{B}(x, y, z, u)=\sigma_{x, y, z} 2 g\left(T^{B}(x, y), T^{B}(z, u)\right)
$$

and so by Formula (3)

$\sigma_{x, y, z} R^{B}(x, y, z, u)=d T^{B}(x, y, z, u)-\sigma_{x, y, z} g\left(T^{B}(x, y), T^{B}(z, u)\right)=\frac{1}{2} d T^{B}(x, y, z, u)$.

Therefore, the Bismut connection satisfies the first Bianchi identity if and only if $g$ is SKT.

Hence, by putting together Theorem 3.1 and Theorem 3.2 we obtain the following

Corollary 3.3. Let $X$ be a complex manifold and let $g$ be a Hermitian metric such that the Bismut connection satisfies the first Bianchi identity. Then,

$$
\nabla^{B} T^{B}=0 \quad \Longleftrightarrow \quad g \text { is } S K T \text {. }
$$

Now we consider some relations with respect to the Levi-Civita connection

Theorem 3.4. Let $X$ be a complex manifold and $g$ a Hermitian metric. If the Bismut connection satisfies the first Bianchi identity and $g$ is SKT then

$$
\nabla^{L C} T^{B}=0
$$

Proof. If the Bismut connection satisfies the first Bianchi identity and $g$ is SKT $\left(d T^{B}=0\right)$, then by Theorem 3.1 we have $\nabla^{B} T^{B}=0$ and so by Formula (3)

$$
0=\left(\nabla_{u}^{B} T^{B}\right)(x, y, z)=\sigma_{x, y, z} g\left(T^{B}(x, y), T^{B}(z, u)\right),
$$

then by [15, Formula (3.18)]

$$
\left(\nabla_{x}^{L C} T^{B}\right)(y, z, u)=\left(\nabla_{x}^{B} T^{B}\right)(y, z, u)+\frac{1}{2} \sigma_{x, y, z} g\left(T^{B}(x, y), T^{B}(z, u)\right)=0 .
$$

Notice that if the dimension of $X$ is 2, then (cf. [15, (2.14)])

$$
T^{B}=-* \theta
$$

hence, on a complex surface

$$
\nabla^{L C} T^{B}=0 \quad \Longleftrightarrow \quad \nabla^{L C} \theta=0 .
$$

Then as a consequence of Theorem 3.4 one has

Corollary 3.5. Let $X$ be a complex surface and $g$ be a Gauduchon metric such that the Bismut connection satisfies the first Bianchi identity. Then, $g$ is Vaisman.

In fact, we can prove that also its converse is true and show Theorem A.

Proof of Theorem A. Since Vaisman metrics are Gauduchon (or equivalently SKT in complex dimension 2), we just need to prove that if $g$ is Vaisman then the Bismut connection satisfies the first Bianchi identity. Recall that on complex surfaces by [1, Appendix A]

$$
\nabla^{B} T^{B}=\nabla^{L C} T^{B}
$$

Since, by hypothesis $\nabla^{L C} \theta=0$ then $\nabla^{B} T^{B}=\nabla^{L C} T^{B}=0$, hence by Theorem 3.2 the Bismut connection satisfies the first Bianchi identity. 
Notice that, up to now we have not used the second part of the definition of Bismut Kähler-like metrics. First of all, notice that if

$$
R^{B}(x, y, z, w)=R^{B}(J x, J y, z, w)
$$

then for the Ricci form of the Bismut connection we have

$$
\rho^{B}(x, y)=\frac{1}{2} \sum R^{B}\left(x, y, e_{i}, J e_{i}\right)=\frac{1}{2} \sum R^{B}\left(J x, J y, e_{i}, J e_{i}\right)=\rho^{B}(J x, J y)
$$

where $\left\{e_{i}\right\}$ denotes an orthonormal basis of the tangent space. Namely, $\rho^{B}$ is a $(1,1)$-form. In particular,

$$
d\left(\rho^{B}\right)^{1,1}=d \rho^{B}=0
$$

where $(\beta)^{1,1}$ denotes the $(1,1)$ component of a 2 -form $\beta$. Hence, since

$$
\left(\rho^{B}\right)^{1,1}=\rho^{C h}+\frac{d J \theta+J d J \theta}{2}
$$

where $\rho^{C h}$ is the Ricci form of the Chern connection, one has that

$$
d d^{c} \theta=0 .
$$

As a consequence,

Proposition 3.6. Let $X$ be a complex surface and let $g$ be a Hermitian metric such that

$$
R^{B}(x, y, z, w)=R^{B}(J x, J y, z, w)
$$

for every tangent vectors $x, y, z, w$. Then, $g$ is locally conformally Kähler.

Proof. Let $\omega$ be the fundamental form associated to $g$. Then, $d \omega=\theta \wedge \omega$ and so we need to prove that $d \theta=0$.

First of all, notice that in any dimension $d \theta$ is a primitive 2 -form, indeed differentiating $d \omega^{n-1}=\theta \wedge \omega^{n-1}$ one has

$$
0=d \theta \wedge \omega^{n-1}=L^{n-1} d \theta .
$$

where $L=\omega \wedge-$ is the operator of multiplication by $\omega$ acting on forms. Therefore, we compute

$$
d^{*} d \theta=-* d(* d \theta)=* J J^{-1} d J d \theta=* J d d^{c} \theta,
$$

where in the second equality we have used for instance [25, Formula (A8)]. Then,

$$
d d^{c} \theta=0 \quad \Longleftrightarrow \quad d^{*} d \theta=0 \quad \Longleftrightarrow \quad d \theta=0 .
$$

3.1. SKT metrics with Lee potential. In [7 a generalization of lck metrics with potential (and so also of Vaisman metrics) is introduced and it is shown that these metrics exist on Calabi-Eckmann manifolds.

Definition 3.7. ([7, 28) A Hermitian manifold $(X, J, g)$ is called LP (or equivalently it has Lee potential) if the (1,0)-part of the Lee form $\theta$ of $g$, namely $\eta:=\theta^{1,0}$, satisfies

$$
\eta \neq 0, \quad \partial \eta=0, \quad \partial \omega=c \eta \wedge \partial \bar{\eta},
$$

for some non-zero constant $c$.

If in addition $\nabla^{B} T^{B}=0$ the metric $g$ is called Generalized Calabi-Eckmann (GCE for short).

As a consequence of Theorem 3.2 the Bismut connection of a Generalized CalabiEckmann SKT metric satisfies the first Bianchi identity.

We show that if a SKT metric is LP then all its powers are $\partial \bar{\partial}$-closed. 
Proposition 3.8. Let $X$ be a complex manifold of complex dimension $n$ endowed with a SKT metric $\omega$ with Lee potential. Then,

$$
\partial \bar{\partial} \omega^{k}=0, \quad \forall 1 \leq k \leq n-1 .
$$

In particular, $\omega$ is $k^{\text {th }}$-Gauduchon for every $1 \leq k \leq n-1$ i.e.,

$$
\partial \bar{\partial}\left(\omega^{k}\right) \wedge \omega^{n-k-1}=0,
$$

and astheno-Kähler, i.e., $\partial \bar{\partial} \omega^{n-2}=0$.

Proof. First of all, notice that for any $k$

$$
\partial \bar{\partial} \omega^{k}=k(\partial \bar{\partial} \omega \wedge \omega-(k-1) \bar{\partial} \omega \wedge \partial \omega) \wedge \omega^{k-2}
$$

Since, $\omega$ is SKT and LP one has that

$$
\partial \bar{\partial} \omega=0, \quad \bar{\partial} \omega \wedge \partial \omega=|c|^{2} \bar{\eta} \wedge \bar{\partial} \eta \wedge \eta \wedge \partial \bar{\eta},
$$

hence

$$
\partial \bar{\partial} \omega^{k}=-k(k-1)|c|^{2}(\bar{\eta} \wedge \bar{\partial} \eta \wedge \eta \wedge \partial \bar{\eta}) \wedge \omega^{k-2} .
$$

Moreover, by the SKT and LP conditions one has also

$$
\partial \eta=0, \quad 0=\partial \bar{\partial} \omega=\bar{c} \partial \bar{\eta} \wedge \bar{\partial} \eta
$$

concluding the proof.

Remark 3.9. Observe that in [28, Remark 2] it was proved that a Hermitian metric that is both SKT and Gauduchon then it is $k^{\text {th }}$-Gauduchon.

The LP assumption is fundamental in the previous Proposition, indeed in the following example we exhibit a manifold with a SKT metric $\omega$ which is not LP and $\partial \bar{\partial} \omega^{n-2} \neq 0$, namely it is not astheno-Kähler.

Example 3.10. Let $(G, J)$ be the 4-dimensional nilpotent Lie group equipped with a left invariant complex structure $J$ with structure equations

$$
\left\{\begin{aligned}
d \varphi^{1} & =0 \\
d \varphi^{2} & =0 \\
d \varphi^{3} & =\lambda_{1} \varphi^{1 \overline{1}}+i a \varphi^{2 \overline{2}} \\
d \varphi^{4} & =\lambda_{2} \varphi^{2 \overline{2}} .
\end{aligned}\right.
$$

with respect to a left invariant unitary coframe $\left\{\varphi^{i}\right\}_{i=1, \ldots, 4}$, where $\lambda_{1}, \lambda_{2}$, a are real numbers and $\lambda_{1}, \lambda_{2}>0$. It is easy to see that left-invariant Hermitian metric $\omega:=\frac{i}{2} \sum_{j=1}^{4} \varphi^{j} \wedge \bar{\varphi}^{j}$ is SKT. Now we show that it is not LP.

By explicit computation one can show that the Gauduchon torsion (1,0)-form $\eta$ is

$$
\eta=\lambda_{2} \varphi^{4}+\left(\lambda_{1}-i a\right) \varphi^{3} .
$$

In particular, computing

$$
\begin{gathered}
\eta \wedge \partial \bar{\eta}=-\lambda_{2}\left(-\lambda_{2}^{2}+i a \lambda_{1}-a^{2}\right) \varphi^{24 \overline{2}}+\lambda_{1}\left(\lambda_{1}+i a\right) \varphi^{14 \overline{1}}-\left(\lambda_{1}-i a\right)\left(-\lambda_{2}^{2}+i a \lambda_{1}-a^{2}\right) \varphi^{23 \overline{2}}+ \\
+\lambda_{1}\left(\lambda_{1}-i a\right)\left(\lambda_{1}+i a\right) \varphi^{13 \overline{1}}
\end{gathered}
$$

and

$$
\partial \omega=-\lambda_{1} \varphi^{13 \overline{1}}+i a \varphi^{23 \overline{2}}-\lambda_{2} \varphi^{24 \overline{2}}
$$

One gets that $\partial \omega=c \eta \wedge \partial \bar{\eta}$ for some non-zero constant $c$ if and only if $\lambda_{1}=-i a=0$ but $\lambda_{1}>0$ so the metric $\omega$ is not LP.

Moreover, notice that the metric $\omega$ is not astheno-Kähler, indeed one has

$$
\partial \bar{\partial} \omega^{2}=2 \lambda_{1} \lambda_{2} \varphi^{123 \overline{1} \overline{2} \overline{4}}+2 \lambda_{1} \lambda_{2} \varphi^{124 \overline{1} \overline{2} \overline{3}} \neq 0 .
$$


3.2. Behavior under small deformations. In this section we partially answer to a question proposed in [4] about the stability of the Bismut Kähler-like property under small deformations of the complex structure. In particular, we show the following

Proposition 3.11. The existence of a SKT metric with Bismut connection satisfying the first Bianchi identity and with $\nabla^{B} T^{B}=0$ on compact complex manifolds is not an open property under small deformations of the complex structure.

Proof. Let $X=\mathbb{S}^{3} \times \mathbb{S}^{3}$ and $\mathbb{S}^{3} \simeq \mathrm{SU}(2)$ be the Lie group of special unitary $2 \times 2$ matrices and denote by $\mathfrak{s u}(2)$ its Lie algebra. Denote by $\left\{e_{1}, e_{2}, e_{3}\right\},\left\{f_{1}, f_{2}, f_{3}\right\}$ a basis of the first copy of $\mathfrak{s u}(2)$, respectively of the second copy of $\mathfrak{s u}(2)$ and by $\left\{e^{1}, e^{2}, e^{3}\right\},\left\{f^{1}, f^{2}, f^{3}\right\}$ the corresponding dual co-frames. Then we have the following commutation relations:

$$
\left[e_{1}, e_{2}\right]=2 e_{3}, \quad\left[e_{1}, e_{3}\right]=-2 e_{2}, \quad\left[e_{2}, e_{3}\right]=2 e_{1},
$$

and the corresponding Cartan structure equations

$$
\left\{\begin{aligned}
d e^{1} & =-2 e^{2} \wedge e^{3} \\
d e^{2} & =2 e^{1} \wedge e^{3} \\
d e^{3} & =-2 e^{1} \wedge e^{2} \\
d f^{1} & =-2 f^{2} \wedge f^{3} \\
d f^{2} & =2 f^{1} \wedge f^{3} \\
d f^{3} & =-2 f^{1} \wedge f^{2}
\end{aligned}\right.
$$

Define a complex structure $J$ on $X$ by setting

$$
J e_{1}=e_{2}, \quad J f_{1}=f_{2}, \quad J e_{3}=f_{3} .
$$

Therefore a complex co-frame of $(1,0)$-forms for $J$ is given by

$$
\left\{\begin{array}{rl}
\varphi^{1} & :=e^{1}+i e^{2} \\
\varphi^{2} & :=f^{1}+i f^{2} \\
\varphi^{3} & :=e^{3}+i f^{3}
\end{array} .\right.
$$

In particular the complex structure equations are given by

$$
\left\{\begin{array}{l}
d \varphi^{1}=i \varphi^{1} \wedge \varphi^{3}+i \varphi^{1} \wedge \bar{\varphi}^{3} \\
d \varphi^{2}=\varphi^{2} \wedge \varphi^{3}-\varphi^{2} \wedge \bar{\varphi}^{3} \\
d \varphi^{3}=-i \varphi^{1} \wedge \bar{\varphi}^{1}+\varphi^{2} \wedge \bar{\varphi}^{2}
\end{array}\right.
$$

Note that $(X, J)$ is a central Calabi-Eckmann threefold and in 26 it is showed that this complex manifold admits a Bismut-flat Hermitian metric, which in particular is SKT and satisfies the first Bianchi identity.

Now let $J_{t}$ be the almost complex structure on $X$ considered in [22] defined as

$$
\left\{\begin{array}{l}
\varphi_{t}^{1}:=\varphi^{1} \\
\varphi_{t}^{2}:=\varphi^{2} \\
\varphi_{t}^{3}:=\varphi^{3}-t \bar{\varphi}^{3}
\end{array} ;\right.
$$

then, using the structure equations (8), a straightforward computation yields to

$$
\left\{\begin{aligned}
d \varphi_{t}^{1} & =\frac{i(\bar{t}+1)}{1-|t|^{2}} \varphi_{t}^{13}+\frac{i(t+1)}{1-|t|^{2}} \varphi_{t}^{1 \overline{3}} \\
d \varphi_{t}^{2} & =\frac{1-\bar{t}}{1-|t|^{2}} \varphi_{t}^{23}+\frac{t-1}{1-|t|^{2}} \varphi_{t}^{2 \overline{3}} \\
d \varphi_{t}^{3} & =i(t-1) \varphi_{t}^{1 \overline{1}}+(t+1) \varphi_{t}^{2 \overline{2}}
\end{aligned}\right.
$$


and consequently $J_{t}$ is integrable. Set $X_{t}=\left(X, J_{t}\right)$, in [22, Remark 3.6] it was proven that when $|t|^{2}+\operatorname{Re} t-\operatorname{Im} t \neq 0$ then $X_{t}$ does not admit any SKT metric. Therefore by Corollary 3.3. for such values of $t, X_{t}$ does not admit any Hermitian metric whose Bismut connection satisfies the first Bianchi identity and with parallel torsion.

\section{Bismut KähleR-LiKe Almost abelian LiE Groups}

In this Section, we study the existence of Hermitian metrics with Kähler-like Bismut connection on simply-connected, almost abelian Lie groups in order to give new examples besides the ones provided in [4] on 6-dimensional solvmanifolds with invariant complex structures, trivial canonical bundle and invariant Hermitian metrics. Let $G$ be a simply-connected, almost abelian Lie group namely, its Lie algebra $\mathfrak{g}$ has a codimension-one abelian ideal $\mathfrak{n}$. In particular, notice that such a $G$ is solvable. Let $(J, g)$ be a left-invariant Hermitian structure on $G$, therefore there exists a basis $\left\{e_{1}, \cdots, e_{2 n}\right\}$ on $\mathfrak{g}$ such that, setting $\mathfrak{n}_{1}=\operatorname{Span}_{\mathbb{R}}\left\langle e_{2}, \cdots, e_{2 n-1}\right\rangle$, one has

$$
\mathfrak{n}=\operatorname{Span}_{\mathbb{R}}\left\langle e_{1}, \cdots, e_{2 n-1}\right\rangle, \quad J e_{1}=e_{2 n}, \quad J\left(\mathfrak{n}_{1}\right) \subset \mathfrak{n}_{1}
$$

By [16] the complex structure $J$ is integrable if and only if ad $e_{2 n}$ leaves $\mathfrak{n}_{1}$ invariant, and $A:=\left.\left(\operatorname{ad} e_{2 n}\right)\right|_{\mathfrak{n}_{1}}$ commutes with $J_{1}:=\left.J\right|_{\mathfrak{n}_{1}}$. Hence one has

$$
\operatorname{ad} e_{2 n}=\left(\begin{array}{ccc}
a & 0 & 0 \\
v & A & 0 \\
0 & 0 & 0
\end{array}\right)
$$

with $a \in \mathbb{R}, v \in \mathfrak{n}_{1}, A \in \mathfrak{g l}\left(\mathfrak{n}_{1}\right)$ and $\left[A, J_{1}\right]=0$.

In particular, we have the following non-trivial Lie brackets

$$
\left[e_{2 n}, x\right]=A x, \quad\left[e_{2 n}, e_{1}\right]=a e_{1}+v
$$

for any $x \in \mathfrak{n}_{1}$.

We fix a real inner product $g$ on $\mathfrak{g}$ with an orthogonal decomposition

$$
\mathfrak{g}=\mathbb{R} e_{1} \oplus \mathfrak{n}_{1} \oplus \mathbb{R} e_{2 n}
$$

and

$$
\mathfrak{n}=\mathbb{R} e_{1} \oplus \mathfrak{n}_{1}
$$

and a compatible integrable complex structure $J$ such that $J e_{1}=e_{2 n}$ and $J\left(\mathfrak{n}_{1}\right) \subset$ $\mathfrak{n}_{1}$.

We recall that by, [5, Lemma 4.2] the metric $g$ is SKT if and only if

$$
a A+A^{2}+A^{t} A \in \mathfrak{s o}\left(\mathfrak{n}_{1}\right) .
$$

In order to study the existence of Bismut Kähler-like metrics we need the expression of the Bismut connection. By using the formula (see [10])

$2 g\left(\nabla_{x}^{B} y, z\right)=g([x, y]-[J x, J y], z)-g([x, z]-[J x, J z], y)-g([y, z]+[J y, J z], x)$,

we get the following 
Lemma 4.1. Let $G$ be an almost abelian Lie group. Then, with the previous notations, the only non-zero components of the Bismut connection are

$$
\begin{aligned}
\nabla_{e_{1}}^{B} e_{1} & =a e_{2 n}, \\
\nabla_{x}^{B} e_{1} & =\sum_{j=2}^{2 n-1} g\left(S(A) J x, e_{j}\right) e_{j}+g(v, x) e_{2 n}, \\
\nabla_{e_{1}}^{B} e_{2 n} & =-a e_{1}, \\
\nabla_{x}^{B} y & =g(x, S(A) J y) e_{1}+g(x, S(A) y) e_{2 n}, \\
\nabla_{e_{1}}^{B} y & =-\sum_{j=2}^{2 n-1} g\left(S(A) J y, e_{j}\right) e_{j}, \\
\nabla_{e_{2 n}}^{B} y & =\frac{1}{2} \sum_{j=2}^{2 n-1} g\left(\left(A-A^{t}\right) y, e_{j}\right) e_{j}, \\
\nabla_{x}^{B} e_{2 n} & =-g(v, x) e_{1}-\sum_{j=2}^{2 n-1} g\left(S(A) x, e_{j}\right) e_{j},
\end{aligned}
$$

with $x, y \in \mathfrak{n}_{1}$ and $S(A):=\frac{1}{2}\left(A+A^{t}\right)$.

In particular if we assume that $A \in \mathfrak{s o}\left(\mathfrak{n}_{1}\right)$ the non-trivial components reduce to

$$
\begin{aligned}
\nabla_{e_{1}}^{B} e_{1} & =a e_{2 n}, \\
\nabla_{x}^{B} e_{1} & =g(v, x) e_{2 n}, \\
\nabla_{e_{1}}^{B} e_{2 n} & =-a e_{1}, \\
\nabla_{e_{2 n}}^{B} y & =A y \\
\nabla_{x}^{B} e_{2 n} & =-g(v, x) e_{1} .
\end{aligned}
$$

If $y \in \mathfrak{n}_{1}$, by explicit computations one gets that that the only non-trivial components of the Bismut curvature tensor are

$$
\begin{aligned}
R^{B}\left(e_{2 n}, y\right) e_{1} & =-g(v, A y) e_{2 n}, \\
R^{B}\left(e_{2 n}, y\right) e_{2 n} & =g(v, A y) e_{1}, \\
R^{B}\left(e_{1}, e_{2 n}\right) e_{1} & =\left(a^{2}+|v|^{2}\right) e_{2 n}, \\
R^{B}\left(e_{1}, e_{2 n}\right) e_{2 n} & =\left(-a^{2}-|v|^{2}\right) e_{1}
\end{aligned}
$$

together with their symmetries. We can now prove

Theorem 4.2. Let $G$ be an almost abelian Lie group and assume that $A \in \mathfrak{s o}\left(\mathfrak{n}_{1}\right)$. Then, the Bismut connection is Kähler-like if and only if Av is g-orthogonal to $\mathfrak{n}_{1}$.

Proof. One can check that the first Bianchi identity holds if and only if $g(v, A y)=0$ for any $y \in \mathfrak{n}_{1}$. Indeed, for instance

$$
R^{B}\left(y, e_{2 n}\right) e_{1}+R^{B}\left(e_{2 n}, e_{1}\right) y+R^{B}\left(e_{1}, y\right) e_{2 n}=g(v, A y) e_{2 n}
$$

and similarly for the other relations. For the $J$-invariance in the first two components of $R^{B}(\cdot, \cdot, \cdot, \cdot)$ one has the same conclusion, for example

$$
R^{B}\left(e_{2 n}, y, e_{1}, e_{2 n}\right)-R^{B}\left(J e_{2 n}, J y, e_{1}, e_{2 n}\right)=-g(v, A y)
$$

and so on. Hence, the Bismut connection is Kähler-like if and only if $g(v, A y)=0$ for every $y \in \mathfrak{n}_{1}$ if and only if $A v$ is $g$-orthogonal to $\mathfrak{n}_{1}$. 
Remark 4.3. Notice that in general these Bismut Kähler-like metrics are not Kähler. More precisely, the torsion of the Bismut connection is given by

$$
\begin{aligned}
T^{B}\left(e_{1}, y\right) & =-g(v, y) e_{2 n}, \\
T^{B}\left(e_{2 n}, y\right) & =g(v, y) e_{1}, \\
T^{B}\left(e_{1}, e_{2 n}\right) & =v
\end{aligned}
$$

for $y \in \mathfrak{n}_{1}$.

And so the only non-zero components of the torsion 3-form are

$$
T^{B}\left(e_{1}, y, e_{2 n}\right)=-g(v, y) .
$$

In particular, one can check explicitly that $\nabla^{B} T^{B}=0$. Hence, these exist explicit examples of metrics that are SKT, with $\nabla^{B} T^{B}=0$ but they do not satisfy the condition $R^{D}(x, y, z, w)=R^{D}(z, y, x, w)$, for every tangent vectors $x, y, z, w$.

Remark 4.4. We notice that having $A \in \mathfrak{s o}\left(\mathfrak{n}_{1}\right)$ is not necessary in order to have a Hermitian metric with Bismut Kähler-like connection on an almost abelian simplyconnected Lie group. Indeed, by [4] the Lie algebra $\mathfrak{h}_{8}$ with structure equations $(0,0,0,0,12)$ is an almost abelian Lie algebra with Bismut Kähler-like connection but the corresponding matrix $A$ is not antisymmetric.

We now discuss the existence of a compact quotient on an explicit example in dimension 6 . First of all notice that if $\mathfrak{g}$ is an almost abelian Lie algebra then it is unimodular (namely all the adjoint maps are traceless) if and only if $a+\operatorname{tr} A=0$. If $A \in \mathfrak{s o}\left(\mathfrak{n}_{1}\right)$, then $\mathfrak{g}$ is unimodular if and only if $a=0$.

Example 4.5. Let $\mathfrak{g}$ be an almost abelian Lie algebra of dimension 6 with non trivial brackets

$$
\left[e_{6}, e_{1}\right]=e_{2}, \quad\left[e_{6}, e_{4}\right]=e_{5}, \quad\left[e_{6}, e_{5}\right]=-e_{4}
$$

Hence we have

$$
\tilde{A}:=\operatorname{ade}_{6}=\left(\begin{array}{ccc}
a & 0 & 0 \\
v & A & 0 \\
0 & 0 & 0
\end{array}\right)
$$

with $v=e_{2}, a=0$ and

$$
A=\left(\begin{array}{cccc}
0 & 0 & 0 & 0 \\
0 & 0 & 0 & 0 \\
0 & 0 & 0 & -1 \\
0 & 0 & 1 & 0
\end{array}\right)
$$

We set $\varphi(t)=e^{t \tilde{A}}$, by 9$] \mathfrak{g}$ admits a lattice if and only if there exists $t_{0} \in \mathbb{R}$ such that $\varphi\left(t_{0}\right)$ is conjugate to an integral matrix. Set $t_{0}:=\pi$, then

$$
\varphi\left(t_{0}\right)=\left(\begin{array}{cccccc}
1 & 0 & 0 & 0 & 0 & 0 \\
\pi & 1 & 0 & 0 & 0 & 0 \\
0 & 0 & 1 & 0 & 0 & 0 \\
0 & 0 & 0 & -1 & 0 & 0 \\
0 & 0 & 0 & 0 & -1 & 0 \\
0 & 0 & 0 & 0 & 0 & 1
\end{array}\right) .
$$

This matrix is conjugate to

$$
\left(\begin{array}{ccccll}
2 & -1 & 1 & 0 & 0 & 0 \\
1 & 0 & 1 & 0 & 0 & 0 \\
0 & 0 & 1 & 0 & 0 & 0 \\
0 & 0 & 0 & -1 & 0 & 0 \\
0 & 0 & 0 & 0 & -1 & 0 \\
0 & 0 & 0 & 0 & 0 & 1
\end{array}\right)
$$


Hence, there exists a lattice $\Gamma$ in $G$ such that $\Gamma \backslash G$ is an almost-albelian solvmanifold of dimension 6 admitting a Bismut Kähler-like Hermitian metric.

In relation to the condition

$$
G^{B}(x, y, z, w):=R^{B}(x, y, z, w)-R^{B}(z, y, x, w)=0,
$$

for every tangent vector $x, y, z, w$. we can prove the following

Theorem 4.6. An almost abelian Lie group $G$ admits a left-invariant SKT metric satisfying (9) if and only if

$$
a=0, \quad v=0, \quad A \in \mathfrak{s o}\left(\mathfrak{n}_{1}\right) .
$$

Proof. We have

$$
\begin{gathered}
G^{B}\left(e_{1}, e_{1}, e_{2 n}, e_{2 n}\right)=-R^{B}\left(e_{2 n}, e_{1}, e_{1}, e_{2 n}\right)=-g\left(R^{B}\left(e_{2 n}, e_{1}\right) e_{1}, e_{2 n}\right)= \\
=-g\left(\nabla_{e_{2 n}}^{B} \nabla_{e_{1}}^{B} e_{1}-\nabla_{e_{1}}^{B} \nabla_{e_{2 n}}^{B} e_{1}-\nabla_{\left[e_{2 n}, e_{1}\right]}^{B} e_{1}, e_{2 n}\right) .
\end{gathered}
$$

Since

$$
\nabla_{e_{1}}^{B} e_{1}=a e_{2 n}, \quad \nabla_{e_{2 n}}^{B} e_{1}=0, \quad \nabla_{e_{2 n}}^{B} e_{2 n}=0
$$

and for every $x \in \mathfrak{n}_{1}$

$$
\nabla_{x}^{B} e_{1}=\sum_{j=2}^{2 n-1} g\left(S(A) J x, e_{j}\right) e_{j}+g(v, x) e_{2 n},
$$

where $S(A):=\frac{1}{2}\left(A+A^{t}\right)$, we have,

$$
G^{B}\left(e_{1}, e_{1}, e_{2 n}, e_{2 n}\right)=a^{2}+\sum_{j=1}^{2 n-2} v_{i}^{2},
$$

hence $G^{B}\left(e_{1}, e_{1}, e_{2 n}, e_{2 n}\right)=0$ if and only if $a=0$ and $v=0$. Similarly, assuming that $a=0$ and $v=0$ we get, for every $x \in \mathfrak{n}_{1}$

$$
G^{B}\left(e_{1}, e_{1}, x, x\right)=\frac{1}{2} \sum_{j=2}^{2 n-1}\left[g\left(S(A) J x, e_{j}\right)\right]^{2}
$$

hence $G^{B}\left(e_{1}, e_{1}, x, x\right)=0$ for every $x \in \mathfrak{n}_{1}$ if and only if $g\left(S(A) J x, e_{j}\right)=0$ for every $x \in \mathfrak{n}_{1}$ and every $j=1, \cdots, 2 n$ (notice that for $j=1$ and $j=2 n$ it is obvious) if and only if $S(A)=0$.

Thus, if $g$ satisfies (9) then we have just proven that $a=0, v=0$, and $A \in \mathfrak{s o}\left(\mathfrak{n}_{1}\right)$. Now we show the viceversa, suppose that $a=0, v=0$ and $A \in \mathfrak{s o}\left(\mathfrak{n}_{1}\right)$ then by similar computations one can show that the only non trivial components of the Bismut connection are, for every $y \in \mathfrak{n}_{1}$

$$
\nabla_{e_{2 n}}^{B} y=\sum_{j=2}^{2 n-1} g\left(A y, e_{j}\right) e_{j}
$$

hence, by definition

$$
R^{B}(x, y) z=\nabla_{x}^{B} \nabla_{y}^{B} z-\nabla_{y}^{B} \nabla_{x}^{B} z-\nabla_{[x, y]}^{B} z=0
$$

for every $x, y, z \in \mathfrak{g}$, hence $R^{B}(x, y, z, w)=0$ for every $x, y, z, w \in \mathfrak{g}$ concluding the proof.

Notice that, in particular, we have proven that an almost abelian Lie group $G$ has $R^{B}(x, y) z=0$ for every $x, y, z \in \mathfrak{g}$ if and only if

$$
a=0, \quad v=0, \quad A \in \mathfrak{s o}\left(\mathfrak{n}_{1}\right),
$$

in particular almost abelian Lie groups satisfying (9) are Bismut flat. In fact, we prove the following 
Theorem 4.7. An almost abelian Lie group $G$ with

$$
v=0 \quad \text { and } \quad A \in \mathfrak{s o}\left(\mathfrak{n}_{1}\right)
$$

is Kähler. In particular, the only almost abelian examples satisfying (9) are Kähler and flat.

Proof. If $v=0$ and $A \in \mathfrak{s o}\left(\mathfrak{n}_{1}\right)$ then the only non-zero commutators become

$$
\left[e_{2 n}, x\right]=A x, \quad\left[e_{2 n}, e_{1}\right]=a e_{1}
$$

where $x \in \mathfrak{n}_{1}$. Hence, computing explicitly the Bismut connection for $(g, J)$ as before one gets that the only non-trivial components are

$$
\nabla_{e_{1}}^{B} e_{1}=a e_{2 n}, \quad \nabla_{e_{1}}^{B} e_{2 n}=-a e_{1}, \quad \nabla_{e_{2 n}}^{B} y=\sum_{j=2}^{2 n-1} g\left(A y, e_{j}\right) e_{j}
$$

where $y \in \mathfrak{n}_{1}$.

Computing explicitly the torsion

$$
T^{B}(x, y)=\nabla_{x} y-\nabla_{y} x-[x, y]
$$

one gets $T^{B}=0$. For instance we compute here, for $y \in \mathfrak{n}_{1}$,

$$
T^{B}\left(e_{2 n}, y\right)=\sum_{j=2}^{2 n-1} g\left(A y, e_{j}\right) e_{j}-\left[e_{2 n}, y\right]=\sum_{j=2}^{2 n-1} g\left(A y, e_{j}\right) e_{j}-A y=0 .
$$

\section{BiSMut-KähleR LiKE CONDITION AND PLURICLOSED FLOW}

Let $(X, J)$ be a complex manifold of complex dimension $n$. In 19 the authors introduce a family of flows called Hermitian curvature flows, among these a particular one is the so called pluriclosed flow which is defined by the equation

$$
\frac{\partial}{\partial t} \omega(t)=-\left(\rho^{B}(\omega)\right)^{1,1}, \quad \omega(0)=\omega_{0}
$$

where $\left(\rho^{B}(\omega)\right)^{1,1}$ denotes the $(1,1)$-part of the Ricci form of the Bismut connection and $\omega_{0}$ is a fixed Hermitian metric. It is easy to see that this flow preserves the SKT condition.

In this section we study the behavior of the Bismut Kähler-like condition under the pluriclosed flow on complex surfaces and on almost abelian Lie groups considered in the previous section.

5.1. Pluriclosed flow on Vaisman surfaces. We recall that a Vaisman metric on a complex manifold $(X, J)$ is a Hermitian metric $\omega$ such that

$$
d \omega=\theta \wedge \omega \text { and } \nabla^{L C} \theta=0,
$$

where $\theta$ is a 1 -form.

In fact, a Vaisman structure on a complex manifold is uniquely determined (up to a positive constant) by its Lee form $\theta$ via the following

$$
\omega=\frac{1}{|\theta|^{2}}(\theta \wedge J \theta-d J \theta) .
$$

On complex dimension 2, Belgun in 6] classified those compact complex surfaces admitting a Vaisman metric, and they are properly elliptic surfaces, primary and secondary Kodaira surfaces, elliptic Hopf surfaces and Hopf surfaces of class 1.

Notice that in particular, on a compact complex surface, Vaisman metrics are SKT. We ask whether the Vaisman condition is preserved along the pluriclosed flow. We answer this question in the case the initial metric has constant scalar curvature.

In order to prove Theorem B we first need the following Lemma. 
Lemma 5.1. Let $X$ be a compact complex surface and let $\omega$ be a Vaisman metric on $X$ with Lee form $\theta$. Then, the Ricci form of the Chern connection is

$$
\rho^{C h}=h d J \theta
$$

for some $h \in \mathcal{C}^{\infty}(X, \mathbb{R})$.

Moreover, the scalar curvature of $\omega$ is constant if and only if $h$ is constant and, in particular, in such a case $c_{1}(X)=0$.

Proof of Theorem B. Let $\left(\omega_{0}, \theta_{0}\right)$ be a Vaisman structure on $X$ with constant scalar curvature; then, by Lemma 5.1, we have

$$
c_{1}(X)=0 \text {. }
$$

Moreover, since $\omega_{0}$ is Vaisman, then

$$
\omega_{0}=\frac{1}{\left|\theta_{0}\right|_{0}^{2}}\left(\theta_{0} \wedge J \theta_{0}-d J \theta_{0}\right)
$$

where with $|\cdot|_{0}$ we denote the norm with respect to $\omega_{0}$ and in particular $\left|\theta_{0}\right|_{0}$ is constant.

We claim that the metrics

$$
\omega_{t}=\frac{1}{\left|\theta_{0}\right|_{0}^{2}}\left(\theta_{0} \wedge J \theta_{0}-f(t) d J \theta_{0}\right),
$$

for a suitable positive function $f(t)$ depending only on $t$ with $f(0)=1$, are Vaisman and they are solutions of the pluriclosed flow.

First of all notice that the corresponding Lee form of $\omega_{t}$ is

$$
\theta_{t}=\frac{1}{f(t)} \theta_{0}
$$

indeed, $d \omega_{t}=\theta_{t} \wedge \omega_{t}$ and clearly $\theta_{t}$ is closed, hence they are locally conformally Kähler.

Moreover, the Lee field $\theta_{t}^{\sharp t}$ with respect to $\omega_{t}$ is

$$
\theta_{t}^{\sharp t}=\frac{1}{f(t)} \theta_{0}^{\sharp 0}
$$

hence by [18, Theorem 1] the metrics $\omega_{t}$ are Vaisman.

In fact, recall that on a compact complex manifold admitting Vaisman metrics, the Lee vector fields of all Vaisman structures are holomorphic, and coincide up to a positive multiplicative constant (cf. [23] and [17]).

Moreover, by a straightforward computation,

$$
\omega_{t}^{2}=f(t)^{2} \omega_{0}^{2}
$$

therefore, the Ricci forms of the Chern connection of $\omega_{t}$ and $\omega_{0}$ must coincide

$$
\rho_{\omega_{t}}^{C h}=\rho_{\omega_{0}}^{C h} .
$$

Hence, the Ricci forms of the Bismut connection of $\omega_{t}$ and $\omega_{0}$ are related by

$$
\rho_{\omega_{t}}^{B}=\rho_{\omega_{t}}^{C h}-d J \theta_{t}=\rho_{\omega_{0}}^{C h}-\frac{1}{f(t)} d J \theta_{0} .
$$

Notice that, since $\omega_{t}$ is lck then $\rho_{\omega_{t}}^{B}$ is a closed $(1,1)$-form.

For the pluriclosed flow we have

$$
\frac{\partial}{\partial t} \omega_{t}=-\frac{1}{\left|\theta_{0}\right|_{0}^{2}} f^{\prime}(t) d J \theta_{0}=d\left(-\frac{1}{\left|\theta_{0}\right|_{0}^{2}} f^{\prime}(t) J \theta_{0}\right)
$$

hence it is necessary to have $c_{1}(X)=0$ to have $\omega_{t}$ solving

$$
\frac{\partial}{\partial t} \omega_{t}=-\left(\rho_{\omega_{t}}^{B}\right)^{1,1}
$$


because we have

$$
d\left(-\frac{1}{\left|\theta_{0}\right|_{0}^{2}} f^{\prime}(t) J \theta_{0}\right)=\rho_{\omega_{0}}^{C h}-d\left(\frac{1}{f(t)} J \theta_{0}\right)
$$

so $\rho_{\omega_{0}}^{C h}$ is an exact form and, up to a constant, it represents the first Chern class of $X$.

Now, by Lemma 5.1

$$
\rho_{0}^{C h}=h d J \theta_{0}
$$

for some constant $h$. Hence,

$$
\rho_{\omega_{t}}^{B}=\left(h-\frac{1}{f(t)}\right) d J \theta_{0} .
$$

and so

$$
-\left(\rho_{\omega_{t}}^{B}\right)^{1,1}=-\rho_{\omega_{t}}^{B}=-\left(h-\frac{1}{f(t)}\right) d J \theta_{0} .
$$

The equation of the pluriclosed flow reduces to find a solution $f(t)$ of

$$
\frac{1}{\left|\theta_{0}\right|_{0}^{2}} f^{\prime}(t) d J \theta_{0}=\left(h-\frac{1}{f(t)}\right) d J \theta_{0}
$$

or equivalently

$$
\left(\frac{1}{\left|\theta_{0}\right|_{0}^{2}} f^{\prime}(t)-h+\frac{1}{f(t)}\right) d J \theta_{0}=0 .
$$

In fact, the equation

$$
\frac{1}{\left|\theta_{0}\right|_{0}^{2}} f^{\prime}(t)-h+\frac{1}{f(t)}=0
$$

admits a unique solution $f(t)>0$ and with $f(0)=1$. Therefore, $\left(\omega_{t}, \theta_{t}\right)$ are Vaisman metrics and solutions of the pluriclosed flow.

Remark 5.2. We notice that, if $X$ is a compact complex surface admitting a Vaisman metric $\omega_{0}$ with constant scalar curvature, then the pluriclosed flow starting with $\omega_{0}$ preserves such a condition. Indeed, by Lemma 5.1 one has for every $t \neq 0$

$$
\rho_{\omega_{t}}^{C h}=h_{t} d J \theta_{t}
$$

for some smooth function $h_{t}$ on $X$, and for $t=0$

$$
\rho_{\omega_{0}}^{C h}=h_{0} d J \theta_{0}
$$

with $h_{0}$ constant by hypothesis.

Then, by the proof of Theorem B we have that

$$
\rho_{\omega_{t}}^{C h}=\rho_{\omega_{0}}^{C h} \quad \text { and } \quad \theta_{t}=\frac{1}{f(t)} \theta_{0}
$$

therefore $h_{t}=f(t) h_{0}$ for every $t$, giving $h_{t}$ constant and so $\omega_{t}$ has constant scalar curvature.

We now give a proof of Lemma 5.1

Proof of Lemma 5.1. Let $\omega$ be a Vasiman metric on $X$. Then, by [2, Lemma 4.4] the Ricci forms of the Bismut and Weyl connections coincide

$$
\rho^{B}=\rho^{W}
$$

where the Weyl connection determined by the Hermitian structure $g$ of $X$ is the unique torsion-free connection $\nabla^{W}$ such that $\nabla^{W} g=\theta \otimes g$. In fact, the Weyl connection is related with the Levi-Civita connection of $g$ via

$$
\nabla_{x}^{W} y=\nabla_{x}^{L C} y-\frac{1}{2} \theta(x) y-\frac{1}{2} \theta(y) x+\frac{1}{2} g(x, y) \theta^{\sharp} .
$$


Since $\rho^{C h}=\rho^{B}+d J \theta=\rho^{W}+d J \theta$ we just need to prove that

$$
\rho^{W}=h d J \theta
$$

for some smooth function $h$ on $X$.

Since the metric is Vaisman then the Ricci tensor $\operatorname{Ric}^{W}$ is symmetric and one has (cf. 3]

$$
\operatorname{Ric}^{W}(x, x)=\operatorname{Ric}^{L C}(x, x)-\frac{1}{2}\left(|\theta|^{2}|x|^{2}-\theta(x)^{2}\right)
$$

where $\operatorname{Ric}^{L C}$ is the Ricci tensor of the Levi-Civita connection.

Therefore, for the Ricci form of the Weyl connection we can compute

$\rho^{W}\left(\theta^{\sharp}, J \theta^{\sharp}\right)=-\operatorname{Ric}^{W}\left(\theta^{\sharp}, \theta^{\sharp}\right)=-\operatorname{Ric}^{L C}\left(\theta^{\sharp}, \theta^{\sharp}\right)+\frac{1}{2}\left(|\theta|^{2}|\theta|^{2}-\theta\left(\theta^{\sharp}\right)^{2}\right)=-\operatorname{Ric}^{L C}\left(\theta^{\sharp}, \theta^{\sharp}\right)$.

Since $\theta$ is parallel with respect to the Levi-Civita connection we have that $R^{L C}\left(x, y, \theta^{\sharp}\right)=0$ for any tangent vectors $x, y$. Hence, $\operatorname{Ric}^{L C}\left(\theta^{\sharp}, \theta^{\sharp}\right)=0$ and so

$$
\rho^{W}\left(\theta^{\sharp}, J \theta^{\sharp}\right)=0 \text {. }
$$

Now assume, without loss of generality, that $|\theta|=1$ and take a orthonormal basis $\theta, J \theta, \xi, J \xi$. Then, we have

$$
\omega=\xi \wedge J \xi+\theta \wedge J \theta, \quad d J \theta=\xi \wedge J \xi,
$$

and

$$
\rho^{W}=h \xi \wedge J \xi+k \theta \wedge J \theta
$$

for some smooth functions $h, k$. Since, $\rho^{W}\left(\theta^{\sharp}, J \theta^{\sharp}\right)=0$, then $k=0$ and so

$$
\rho^{W}=h \xi \wedge J \xi=h d J \theta .
$$

Now, notice that the scalar curvature $s$ of $g$ is constant if and only if the same holds for the trace $b$ of $\rho^{B}$. This follows by [2, Formula (2.12)],

$$
b=s-2|\theta|^{2}+\frac{1}{2}|d \omega|^{2}
$$

having $|d \omega|^{2}=|\theta|^{2}$ constant for a Vaisman metric on a complex surface. Therefore, $b$ is constant if and only if the same holds for the function $h$, indeed

$$
b=\rho^{B}\left(J \xi^{\sharp}, \xi^{\sharp}\right)+\rho^{B}\left(J\left(J \xi^{\sharp}\right), J \xi^{\sharp}\right)+\rho^{B}\left(J \theta^{\sharp}, \theta^{\sharp}\right)+\rho^{B}\left(J\left(J \theta^{\sharp}\right), J \theta^{\sharp}\right)=-2 h,
$$

where we have used that $\rho^{B}=\rho^{W}=h \xi \wedge J \xi$.

5.2. Pluriclosed Flow on almost abelian Lie groups. In [5] it is shown that the pluriclosed flow on almost abelian Lie algebras reduces to

$$
\left\{\begin{aligned}
a^{\prime} & :=c a \\
v^{\prime} & :=c v+S v-\frac{1}{2}|v|^{2} v ; \\
A^{\prime} & :=c A
\end{aligned}\right.
$$

where

$$
c=\left(\frac{k}{4}-\frac{1}{2}\right) a^{2}-\frac{1}{2}|v|^{2} \in \mathbb{R}, \quad 2 k=\operatorname{rk}\left(A+A^{t}\right)
$$

and

$$
S=\left(\frac{k}{4}-\frac{1}{2}\right) a^{2} \operatorname{Id}_{\mathfrak{n}_{1}}-\frac{1}{2} A A^{t}+\frac{a}{4}\left(A+A^{t}\right) .
$$

We prove the following

Theorem 5.3. Let $G$ be a simply-connected almost abelian Lie group. If $A_{0} \in$ $\mathfrak{s o}\left(\mathfrak{n}_{1}\right)$, then the pluriclosed flow preserves the Bismut Kähler-like condition. 
Proof. Notice that if $A_{0} \in \mathfrak{s o}\left(\mathfrak{n}_{1}\right)$ then by uniqueness of the solution $A(t) \in \mathfrak{s o}\left(\mathfrak{n}_{1}\right)$. Hence, if we assume $A_{0} \in \mathfrak{s o}\left(\mathfrak{n}_{1}\right)$ one gets

and

$$
c=-\frac{1}{2} a^{2}-\frac{1}{2}|v|^{2}, \quad k=0
$$

$$
S=-\frac{1}{2} a^{2} \operatorname{Id}_{\mathfrak{n}_{1}}+\frac{1}{2} A^{2} .
$$

We show that if $A_{0} \in \mathfrak{s o}\left(\mathfrak{n}_{1}\right)$ and at $t=0$ the metric is Bismut Kähler-like then it remains Bismut Kähler-like along the flow. Indeed, if if $A_{0} \in \mathfrak{s o}\left(\mathfrak{n}_{1}\right)$ and at $t=0$ the metric is Bismut Kähler-like one has that $A_{0} v_{0}$ is orthogonal to $\mathfrak{n}_{1}$ and by [5] $\mathfrak{n}_{1}$ is preserved along the flow. We compute, for any $y \in \mathfrak{n}_{1}$

$$
\begin{gathered}
\frac{d}{d t}(g(v, A y))=g\left(v^{\prime}, A y\right)+g\left(v, A^{\prime} y\right)=g\left(c v+S v-\frac{1}{2}|v|^{2} v, A y\right)+c g(v, A y)= \\
=2 c g(v, A y)-\frac{1}{2} a^{2} g(v, A y)+\frac{1}{2} g\left(A^{2} v, A y\right)-\frac{1}{2}|v|^{2} g(v, A y)= \\
=3 c g(v, A y)+\frac{1}{2} g\left(A^{2} v, A y\right)=g\left(v, A\left(3 c \operatorname{Id}_{\mathfrak{n}_{1}}+\frac{1}{2} A^{2}\right) y\right)
\end{gathered}
$$

hence

$$
\frac{d}{d t}(g(A v, y))=g\left(A v,\left(3 c \operatorname{Id}_{\mathfrak{n}_{1}}+\frac{1}{2} A^{2}\right) y\right)
$$

Then, if $A_{0} v_{0}$ is orthogonal to $\mathfrak{n}_{1}$ by uniqueness of the solution it remains orthogonal along the flow and so the Bismut Kähler-like condition is preserved.

\section{REFERENCES}

[1] I. Agricola, A. C. Ferreira, Einstein manifolds with skew torsion, Q. J. Math. 65 (2014), no. $3,717-741$

[2] B. Alexandrov, S: Ivanov, Vanishing theorems on Hermitian manifolds, Differential Geom. Appl. 14 (2001), no. 3, 251-265.

[3] B. Alexandrov, S. Ivanov, Weyl structures with positive Ricci tensor, Differential Geom. Appl. 18 (2003), no. 3, 343-350.

[4] D. Angella, A. Otal, L. Ugarte, R. Villacampa, On Gauduchon connections with Kähler-like curvature, to appear in Commun. Anal. Geom., arXiv:1809.02632 [math.DG], 2018

[5] R. M. Arroyo, R. Lafuente, The long-time behavior of the homogeneous pluriclosed flow, Proc. London Math. Soc. (3) 119 (2019), 266-289.

[6] F. A. Belgun, On the metric structure of non-Kähler complex surfaces, Math. Ann. 317 (2000), 1-40.

[7] F. A. Belgun, On the metric structure of some non-Kähler complex threefolds, arXiv: 1208.4021

[8] J.-M. Bismut, A local index theorem for non-Kähler manifolds. Math. Ann. 284 (1989), no. 4, 681-699.

[9] C. Bock, On low-dimensional solvmanifolds, Asian J. Math, 20 (2016), no. 2, 199-262.

[10] I. Dotti, A. Fino, HyperKähler torsion structures invariant by nilpotent Lie groups. Classical Quantum Gravity 19 (2002), no. 3, 551-562.

[11] A. Fino, A. Otal, L. Ugarte, Six-dimensional solvmanifolds with holomorphically trivial canonical bundle, Int. Math. Res. Not. IMRN (2015), no. 24, 13757-13799.

[12] A. Fino, M. Parton, S. Salamon, Families of strong KT structures in six dimensions, Comment. Math. Helv. 79, no. 2, 317-340 (2004)

[13] P. Gauduchon, La 1-forme de torsion d'une variété hermitienne compacte, Math. Ann. 267 (1984), no. 4, 495-518.

[14] P. Gauduchon, Hermitian connections and Dirac operators, Boll. Un. Mat. Ital. B (7) 11 (1997), no. 2, suppl., 257-288.

[15] S. Ivanov, G. Papadopoulos, Vanishing theorems and string backgrounds, Classical and Quantum Gravity, textbf18 (2001), no. 6, 1089-1110.

[16] J. Lauret, E. A. R. Valencia, On the Chern-Ricci flow and its solitons for Lie groups, Math. Nachr. 288 (2015) 1512-1526.

[17] F. Madani, A. Moroianu, M. Pilca, LcK structures with holomorphic Lee vector field on Vaisman-type manifolds, arXiv:1905.07300 [math.DG], 2019. 
[18] A. Moroianu, S. Moroianu, L. Ornea, Locally conformally Kähler manifolds with holomorphic Lee field, Differential Geom. Appl. 60 (2018), 33-38.

[19] J. Streets and G. Tian, A parabolic flow of pluriclosed metrics, Int. Math. Res. Not. 16 (2010), 3101-3133.

[20] J. Streets, G. Tian, Regularity theory for pluriclosed flow. C. R. Math. Acad. Sci. Paris 349 (2011), no. 1-2, 1-4.

[21] J. Streets, G. Tian, Regularity results for pluriclosed flow. Geom. Topol. 17 (2013), no. 4, 2389-2429.

[22] N. Tardini, A. Tomassini, On geometric Bott-Chern formality and deformations, Ann. Mat. Pura Appl. (4) 196 (2017), no. 1, 349-362.

[23] K. Tsukada, Holomorphic forms and holomorphic vector fields on compact generalized Hopf manifolds, Compositio Mathematica, 93 (1) (1994), 1-22

[24] L. Ugarte, Hermitian structures on six-dimensional nilmanifolds, Transform. Groups 12 (2007), no. 1, 175-202.

[25] I. Vaisman, On some variational problems for 2-dimensional Hermitian metrics, Ann. Global Anal. Geom. 8 (1990), no. 2, 137-145.

[26] Q. Wang, B. Yang, F. Zheng, On Bismut flat manifolds, arXiv:1603.07058 [math.DG], 2016

[27] B. Yang, F. Zheng, On curvature tensors of Hermitian manifolds, arXiv:1602.01189, to appear in Comm. Anal. Geom.

[28] S. T. Yau, Q. Zhao, F. Zheng, On Strominger Kähler-like manifolds with degenerate torsion, arXiv:1908.05322 [math.DG].

[29] Q. Zhao, F. Zheng, Complex nilmanifolds and Kähler-like connections, arXiv:1904.09707 [math.DG].

[30] Q. Zhao, F. Zheng, Strominger connection and pluriclosed metrics, arXiv:1904.06604 [math.DG].

(Anna Fino) Dipartimento di Matematica "G. Peano”, Università degli studi di Torino, Via Carlo Alberto 10, 10123 Torino, Italy

E-mail address: annamaria.fino@unito.it

(Nicoletta Tardini) Dipartimento di Matematica "G. Peano", Università degli studi di Torino, Via Carlo Alberto 10, 10123 Torino, Italy

E-mail address: nicoletta.tardini@gmail.com

Current address: Dipartimento di Scienze Matematiche, Fisiche e Informatiche, Unità di Matematica e Informatica, Università degli Studi di Parma, Parco Area delle Scienze 53/A, 43124 Parma, Italy 